\title{
Elevation pattern analysis of common passive bistatic radar illuminators of opportunity
}

\author{
D. W. O'Hagan, H. D. Griffiths, S. M. Ummenhofer and S. G. Paine
}

\begin{abstract}
In this paper the elevation coverage of common passive radar illuminators of opportunity is evaluated. In constructing radar performance metrics, it is imperative to include the radiation characteristics of the source, both in azimuth and elevation. Many passive radar performance studies have only considered one plane, the azimuth, neglecting to incorporate the elevation plane in models. The non-inclusion of elevation patterns has primarily been due to lack of precise details of their specification and nature. The authors have consulted the broadcast antenna manufacturing industry and present here transmitter elevation patterns for the three prevalent passive radar illuminators of opportunity, FM radio, DAB, and DVB-T. The patterns presented are typical of broadcast patterns in use throughout the world. The patterns have already been used by a number of national research institutes for more accurate passive radar system modelling. It is shown that airborne targets frequently reside outside of the elevation mainlobe, which has direct consequences for target detection performance.
\end{abstract}

Index Terms-Passive Bistatic Radar (PBR), beam-tilt, elevation mainlobe, broadcast illuminators of opportunity, half power beamwidth (HPBW).

\section{INTRODUCTION}

$\mathrm{O}$ $\mathrm{NE}$ of the primary objectives in broadcast engineering is to achieve wide-area coverage with adequate field strength so that users can receive the transmissions of interest. The 'users' in terms of broadcast engineering considerations are ground-based receivers for such services as FM radio, TV, DAB, DVB-T and GSM.

For common passive radar illuminators of opportunity, namely FM radio, DAB and DVB-T, the field strength at a given distance from the antenna depends on ERP, antenna heights, local terrain and tropospheric scattering conditions. The antenna systems usually consist of several individual radiating bays fed as a phased array. The radiation characteristics of the broadcast antenna may be concentrated in both the horizontal (azimuth) and vertical (elevation) planes. The azimuth pattern is often approximately omnidirectional, but the elevation pattern is usually highly

Manuscript received June XX, 2016

D. W. O'Hagan and S. G. Paine are with the University of Cape Town. H. D. Griffiths is with University College London. M. Ummenhofer is with Fraunhofer FHR.

UCT Dept. of Electrical Eng. (phone: +27 (0)21 650 2919; e-mail: daniel.ohagan@uct.ac.za). directional, with the energy directed towards the population to be served, minimising the radiation out into space. Reducing radiation into space (i.e., beyond the horizon) requires the elevation radiation pattern to be tilted slightly below the horizon. This is a common procedure in broadcast engineering and is referred to as beam-tilt. The radar/radio (source) horizon is shown in Fig. 1.

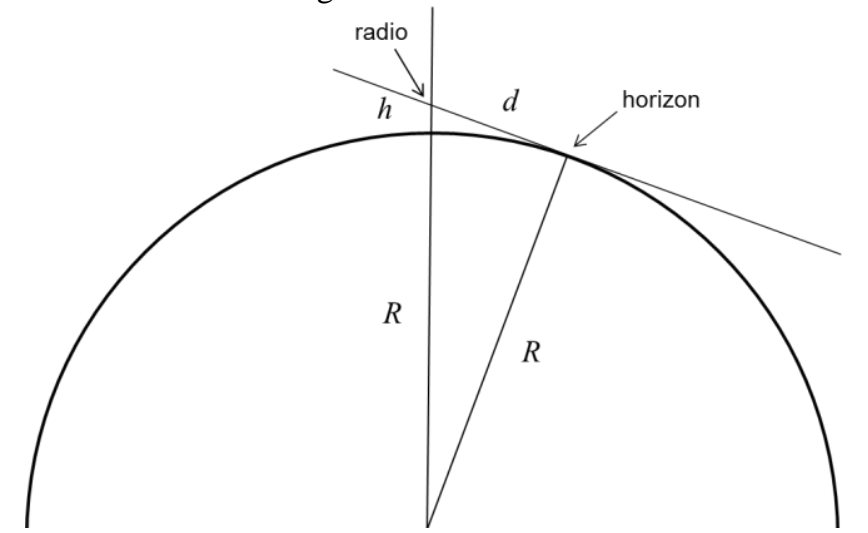

Fig. 1. The radio horizon is limited by the curvature of the Earth. Broadcast antennas tilt their elevation lobe below the actual horizon to avoid wasting power beyond the horizon. In the figure, $R$, is the radius of the Earth.

Beam-tilt and narrow elevation mainlobes impact on the detection capabilities of bistatic radars that use broadcast illuminators of opportunity. Passive radar performance limitations resulting from beam-tilt and pattern shaping ${ }^{1}$ are most likely to be observed when trying to detect targets at higher altitudes and at ranges closer to the transmitter. For example, civilian jet-liners cruising at altitudes of about $10 \mathrm{~km}$ may be outside of the elevations mainlobe illumination. Fig. 2 is an illustrative example of a PBR using an illuminator of opportunity. The example shows the target of interest at an altitude to place it above the narrow elevation mainlobe and about to traverse the sidelobes.

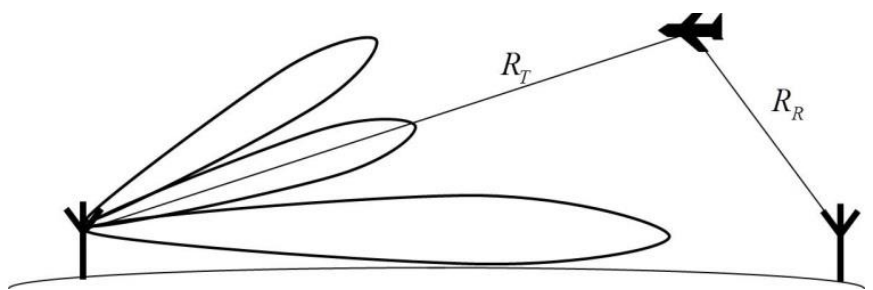

1 Elevation mainlobe shaping refers to a narrowing of the mainlobe elevation beamwidth. The beamwidth can be narrowed, or squeezed, by increasing the number of radiating-bays, or levels, of the broadcast antenna. Each element is stacked and fed as in a phased array configuration to achieve the narrowing of the elevation mainlobe. 
Fig. 2. Illustration of a bistatic radar using an illuminator of opportunity with a narrow and down-tilted elevation mainlobe.

\section{BEAM-TILT AND ELEVATION MAINLOBE SHAPING AND} THEIR IMPACT ON THE DETECTION PERFORMANCE OF CRUISINGAND HIGH-ALTITUDE TARGETS

The FM radio, DAB, and DVB-T broadcasts are typically limited to line-of-sight ranges. Therefore, coverage is localised to a range of about $120 \mathrm{~km}$ from the transmitter [1], which amounts to a transmitter station height, including mast height, in excess of approximately $500 \mathrm{~m}$ above sea level and a receiver station height of $50 \mathrm{~m}$.

At RF frequencies the Earth and its atmosphere act in a similar manner to a waveguide and bend RF energy downwards due to the reduction of atmospheric refractive index with height. Empirical measurements in the 1940s revealed that a correction factor of 1.33 (or 4/3) was a reasonable approximation for the Earth's radius to account for wave bending at RF frequencies [2]. The expression for calculating the radar horizon, which includes the $4 / 3$ compensation factor, is:

$$
\begin{aligned}
& d=\sqrt{2 \times h \times \frac{4}{3} \times R} \\
& d=130 \sqrt{h}
\end{aligned}
$$

where

$$
\begin{aligned}
d= & \text { distance to the radio horizon }(\mathrm{km}) ; \\
h= & \text { height of transmitter site including antenna mast } \\
& \text { height }(\mathrm{m}) ; \\
R= & \begin{array}{l}
\text { radius of the Earth, which at the equator is } \\
\text { approximately } 6378 \mathrm{~km} .
\end{array}
\end{aligned}
$$

Fig. 3 shows a plot of the radar horizon calculated using (1) for a range of transmitter heights from 1 to $600 \mathrm{~m}$.

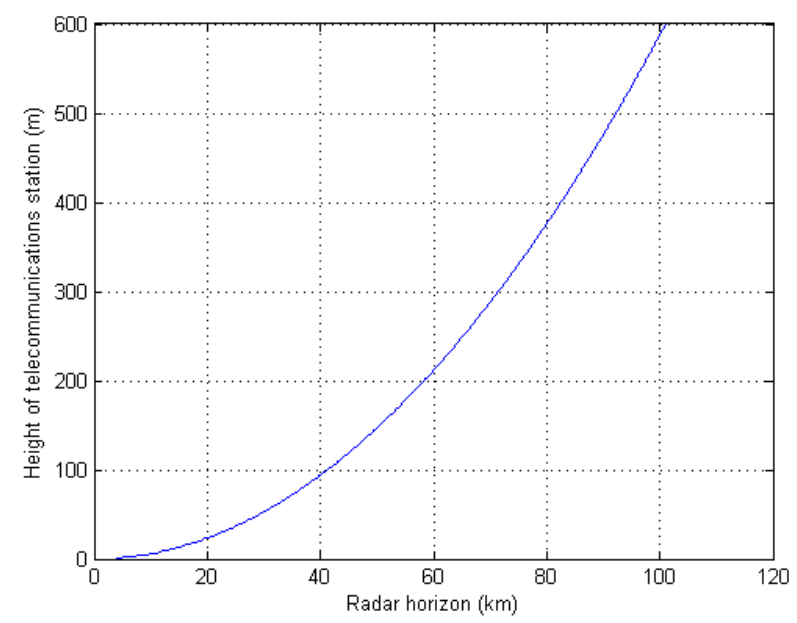

Fig. 3. Radar horizon for a range of transmitter site heights (including mast height) from $1 \mathrm{~m}$ to $600 \mathrm{~m}$.

In the context of radars detecting targets at altitudes, the radar horizon is obviously extended as a function of the target height. The radar horizon is then $d=130\left(\sqrt{h_{T}}+\sqrt{h_{t}}\right)$ where $h_{T}$ is the transmitter height and $h_{t}$ is the target altitude.

Civilian passenger-jet aircraft cruise at altitudes of about $30,000 \mathrm{ft}$. to $40,000 \mathrm{ft}$. (approximately $9 \mathrm{~km}-12 \mathrm{~km}$ ). Cruising altitude and speed are chosen to optimise flight performance, and when cruising, an aircraft is most fuel efficient. Factors that determine cruise altitude include the type and condition of the aircraft, payload weight, centre of gravity, air temperature, humidity and flight speed [3-6].

Military fighter-jet aircraft when not in survival or combat mode can cruise at altitudes of $40,000 \mathrm{ft}$. or higher (i.e. approximately $12 \mathrm{~km}$ and higher). Tier II plus high altitude long endurance UAVs (Unmanned Aerial Vehicles) can reach altitudes of $60,000 \mathrm{ft}$. to $65,000 \mathrm{ft}$. (approximately $20 \mathrm{~km}$ !) [3 $-6]$.

It is obvious, therefore, that radars relying on transmitters with vertical beam down-tilt and narrow elevation beamwidths (as well as non-radar waveforms) are not an ideal choice for the detection of some very high altitude targets. More specifically, the elevation tilt is advantageous for illuminating targets as they first appear over the horizon at far range. If a target were to remain at constant flight-level, say cruising altitudes, then as its ground range to the transmitter decreases, it will migrate out of the elevation mainlobe illumination.

There have been, numerous demonstrations of passive radars detecting and tracking civil passenger-jet aircraft at altitudes of 30,000 ft. to 40,000 ft. (e.g. [7 - 11]), but in these instances, the targets were typically at far range from the transmitter.

When contemplating using a passive radar for a particular function, punctilious thought should be given to the full altitude range of the primary targets of interest. The desired target's upper altitude extremity should then be consolidated with the most appropriate elevation pattern. These are exactly the considerations that led to the $\operatorname{cosec}^{2}$ elevation pattern of most Primary Surveillance Radars (PSR).

From here on cruising altitude will refer to aircraft that are at 30,000 ft. $(9 \mathrm{~km})$ and higher.

\section{CONCEPT OF RADAR ELEVATION COVERAGE}

To achieve radar coverage of the higher elevation airspace, especially at close range to the radar, many radars such as Primary Surveillance Radars (PSRs) use a $\operatorname{cosec}^{2}$ elevation pattern. The purpose of the $\operatorname{cosec}^{2}$ pattern is to give a target echo strength that is independent of target range, for a given target altitude. This reduces the dynamic range requirement of the radar receiver. A typical example of the $\operatorname{cosec}^{2}$ pattern is shown in Fig. 4 as specified for the Indra S-Band PSR [12]. The $\operatorname{cosec}^{2}$ pattern permits illumination of cruising altitude aircrafts and such PSRs are designed to a specific specification like CAP 670 for Air Traffic Services [13]. 


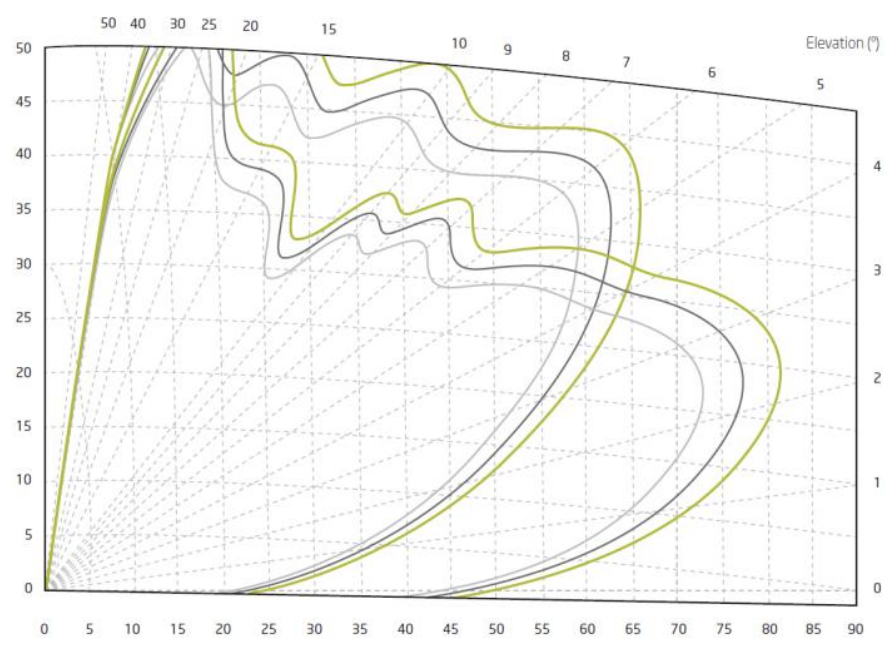

Fig. 4. A high-beam and low-beam $\operatorname{cosec}^{2}$ elevation pattern specified for the Indra S-Band PSR.

It can be seen from Fig. 4 that the $\operatorname{cosec}^{2}$ PSR elevation patterns meets its specification of providing a fairly flat, high altitude, coverage pedestal from near-range to the radar out to its documented range. It is important to observe that the $\operatorname{cosec}^{2}$ pattern has limited coverage for low altitude targets. In Fig. 4, for a target altitude of $5^{\circ}$ it would only become illuminated when it is approximately $40 \mathrm{nmi}$ from the radar. Therefore, PSRs employing $\operatorname{cosec}^{2}$ elevation patterns have modest lowaltitude target detection capabilities.

In contrast most broadcast services such as $\mathrm{FM}$ radio, $\mathrm{DAB}$ and DVB have much narrower elevation coverage and are actually electrically tilted in elevation to avoid radiating energy wastefully beyond the horizon. Passive radar that utilise broadcast illuminators of opportunity may have superior ability over PSRs to provide coverage of the lower altitude airspace. A general performance comparison of PSR and Passive Radar will not be provided here as the two sensors are significantly different - our focus is only on elevation coverage characteristics.

Fig. 5 is a FEKO simulated eight-element (8-bay), verticallystacked, FM radio broadcast antenna. There is a successive phase shift between elements to cause a narrowing in the width of the elevation mainlobe. The phasing also results in electrical beam-tilts of about $1^{\circ}$. Further beam-tilt can be achieved by mechanically adjusting the panel so that it is mounted at a given depression angle, but the dominant technique is electrical tilt. It is even possible, indeed often routine, to alter the electrical tilt remotely.

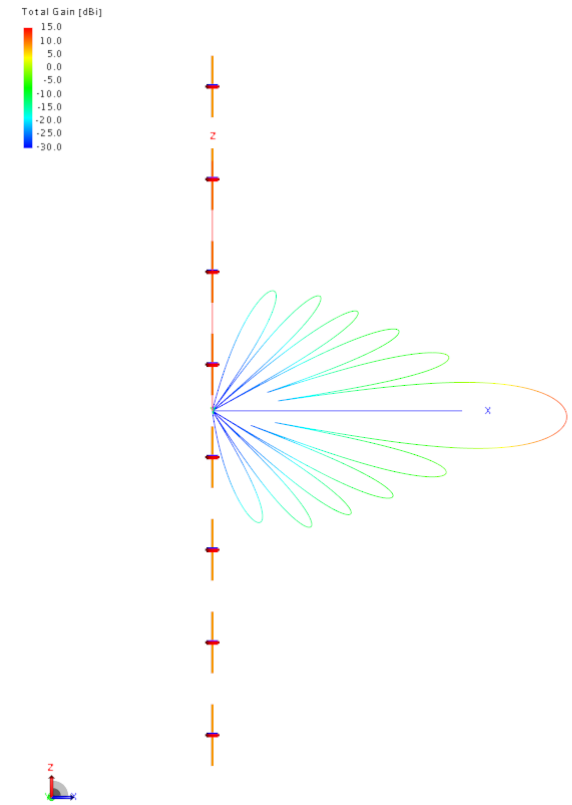

Fig. 5. FEKO simulation of an 8-bay FM broadcast antenna. Only the elevation pattern is shown. The beam-tilt angle is $1^{\circ}$ below the horizon.

Fig. 6 shows the FEKO simulated 3-D pattern for the 8-bay broadcast antenna just described.

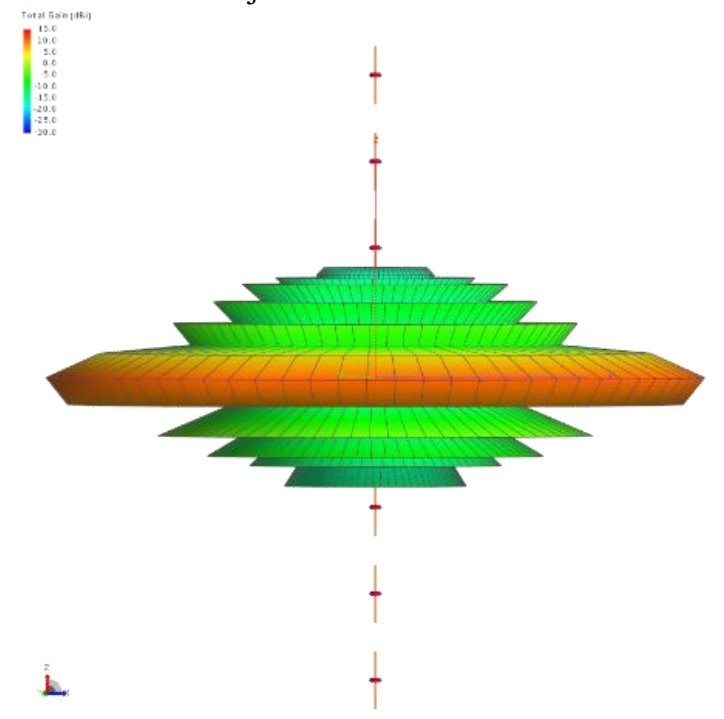

Fig. 6. FEKO simulation of an 8-bay FM broadcast antenna. This is the 3-D pattern with an approximate azimuth profile and an elevation beam-tilt of $1^{\circ}$.

The extent of power radiated above the horizon, considering only the 1-D elevation pattern, can be determined from (2):

$P_{\text {above horizon }} \approx \frac{\int_{1}^{+\pi / 2} G(\phi) d \phi}{\int_{-\pi / 2}^{+\pi / 2} G(\phi) d \phi} \times P_{\text {total_el }}$

where $P_{\text {total } e l}$ is the total power available in the elevation extent and $\phi$ is the elevation angle. The power radiated by the antenna in any given direction is:

$$
P(\theta, \phi)=\int_{0}^{2 \pi} \int_{0}^{\pi / 2} P(\theta, \phi) \sin \phi d \theta d \phi \times T P R
$$


where $T P R$ is the total power radiated by the antenna and $\iint_{4 \pi} P(\theta, \phi) \sin \phi d \theta d \phi$ in stradians is the beam solid angle.

vertical patterns in Fig. 12, where the first three nulls below the horizon have been filled. This can be compared to Fig.5, where the deep nulls in the EM model indicate uniform phase progression, so there is no null-fill, or sidelobe asymmetry about the main lobe $[14,15]$.

From (2) it is typical that a broadcast antenna with a $1^{\circ}$ beamtilt would radiate about $40 \%$ of its total power available in the elevation extent (i.e., $>0.4 P_{\text {total_el }}$ ) above the horizon.

\section{BROADCAST ELEVATION PATTERNS USED FOR THIS RESEARCH}

The RFS Company (Radio Frequency Systems) is a dominant leading global company in broadcast-antenna engineering and design, construct and install broadcast towers for customers worldwide. RFS has provided the elevation patterns on which this research is based. They possess a very heavy duty pattern-measurement rotator designed to take a full column of a customer's antenna mounted vertically. Sometimes, only a section is used, especially for arrays that have many panels around the column. The azimuth pattern is measured on the far-field pattern test range, and this forms part of the factory acceptance test for each customer.

Clearly given the scale of some antennas, it is not an easy task to measure the full elevation pattern of very long and heavy arrays, as it is not possible to mount them horizontally on a rotator. So the elevation patterns are usually calculated using sophisticated EM modelling software. To produce accurate pattern models, the software uses, as a building-block, a measured elevation pattern of a panel immersed in an array environment (a very short array). The accuracy of this approach has been confirmed by full electromagnetic modelling and tests that included sampling of near-fields in a large anechoic chamber followed by far-field pattern generation. Further confirmation to validate the accuracy of patterns is also sometimes obtained from customers' helicopter pattern measurements of installed arrays and their coverage measurements.

Prior to delivery, the full antenna is usually mounted vertically on a stand, using a crane, together with its full feeding network. Test signals are injected into the inputs using a network analyser, and VSWR is measured. Each panel on the array has a sampling port next to its input, and the measured phases of voltages at the panel inputs are compared with those used in the generation of the elevation pattern. Experience has shown that if the voltages at the panels have the correct phase, the calculated elevation patterns will be accurate [14].

As well as providing down-tilt in the pattern to achieve enhanced coverage, it is normal to include null-fill of the first few nulls below the main lobe to avoid any deep nulls that will cause rings of poor coverage close to the transmitter. Null-full is commonly achieved by using a perturbation of the vertical phase distribution only, with each level of panels delivering equal power. This is what produces the asymmetry in the

\section{FM RADIO ELEVATION COVERAGE}

Fig. 7 shows an elevation pattern of a 6-bay FM radio broadcast antenna. It is usual for broadcast antenna manufacturers to provide radiation patterns in terms of relative normalised field strength $\left(\mathrm{E} / \mathrm{E}_{\max }\right)$.

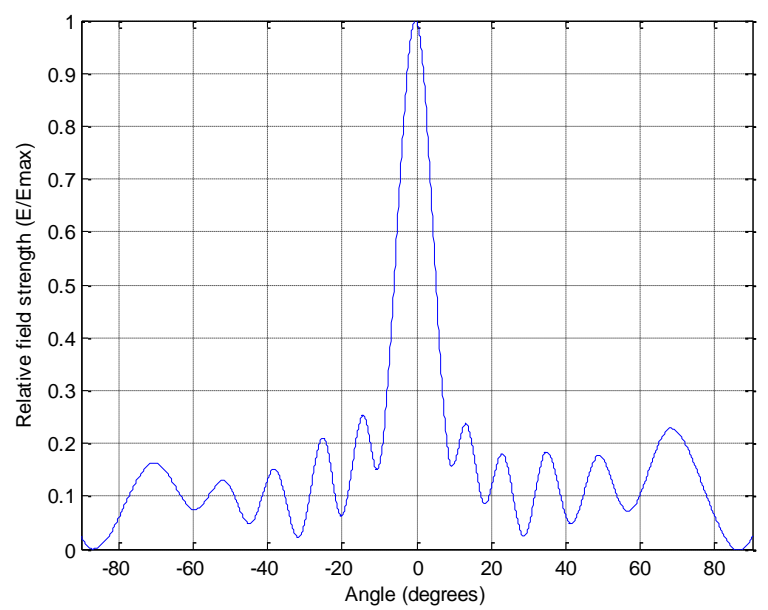

Fig. 7. Elevation pattern of a 6-bay FM radio transmit antenna. In this case the beam-tilt angle is $1.1^{\circ}$ with a directivity of $8.02 \mathrm{dBd}$. Note that $-90^{\circ}$ is directed to the nadir and $90^{\circ}$ is directed to the zenith.

In Fig. 7, the elevation HPBW (or $0.707 \mathrm{~V} / \mathrm{m}$ relative field strength) is $9^{\circ}$. In the absence of beam-tilt, half of the $9^{\circ}$ beamwidth would be above the $0^{\circ}$ line. However, in this case the beam-tilt is $1.1^{\circ}$ and so the angular width of the mainlobe above the horizon line is $3.4^{\circ}$ at the elevation HPBW point.

It is often more informative for the radar engineer to consider the equivalent relative power plot. For example, in Fig. 8 it is easy to see that the first sidelobe occurs at approximately $12.3^{\circ}$ and is about $12 \mathrm{~dB}$ down from the main peak. It is also worth observing that the pattern sidelobes are not symmetric.

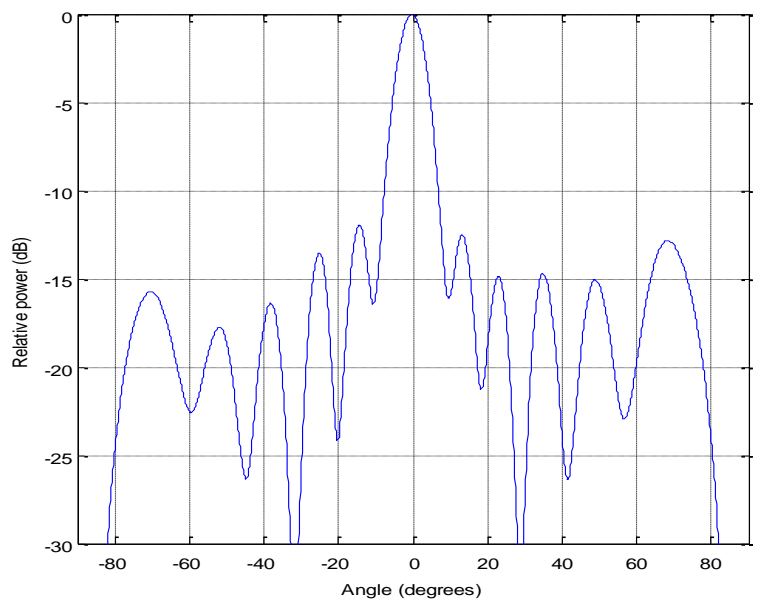

Fig. 8. Elevation pattern of a 6-bay FM radio transmit antenna in log-scale. 
Antenna lobing effects may result in acute signal-to-noise ratio fluctuations at the receiver. The lobe characteristics of the FM radio 6-bay broadcast antenna is shown in Fig. 9 in the elevation plane to illustrate its extent.

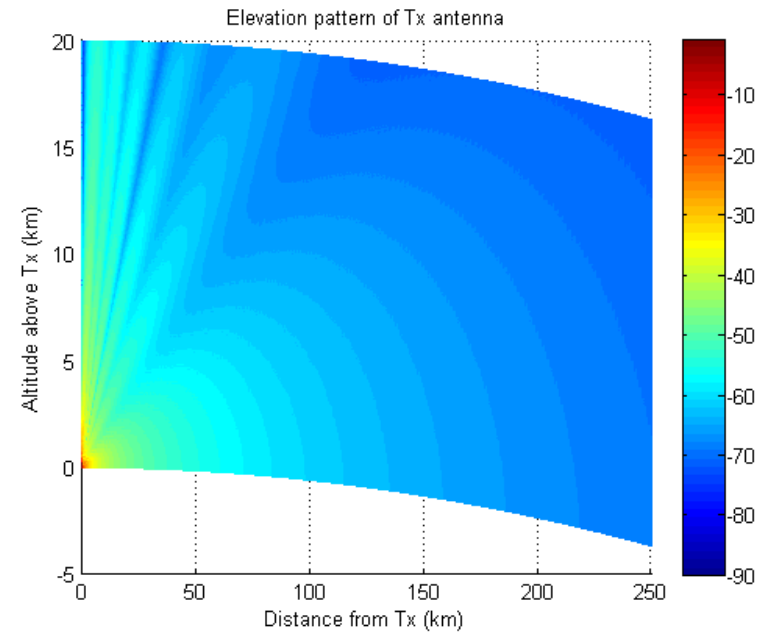

Fig. 9. Illustrating the extent of the elevation pattern lobes of a 6-bay FM radio transmit antenna.

Fig. 9 includes the $1.1^{\circ}$ beam-tilt angle and is plotted for an arbitrary transmit power $\left(P_{T}\right)$ of $10 \mathrm{~kW}$. The transmit power of $10 \mathrm{~kW}$ is plausible and realistic, but arbitrary because the purpose is to demonstrate the elevation pattern characteristics and not to calculate the usual detection SNR values. The pattern is plotted over $250 \mathrm{~km}$ range, which includes the $4 / 3$ Earth compensation factor. The curvature of the smooth Earth is visible; however, in reality VHF signals will exhibit very limited over-the-horizon capability. The antenna height is assumed negligible with respect to target altitudes and is located at $(0,0)$. Since the radio horizon of typical broadcast antennas rarely exceeds about $120 \mathrm{~km}$ and since the detection ranges of FM-based PBRs have seldom been consistently demonstrated beyond $100 \mathrm{~km}$, the distance from the transmitter will therefore be restricted to $100 \mathrm{~km}$. This can be seen in Fig. 10, which in this case does not include Earth curvature (i.e. the Earth is assumed flat to $100 \mathrm{~km}$ from the transmitter). The plot does, however, include the beam-tilt effect.

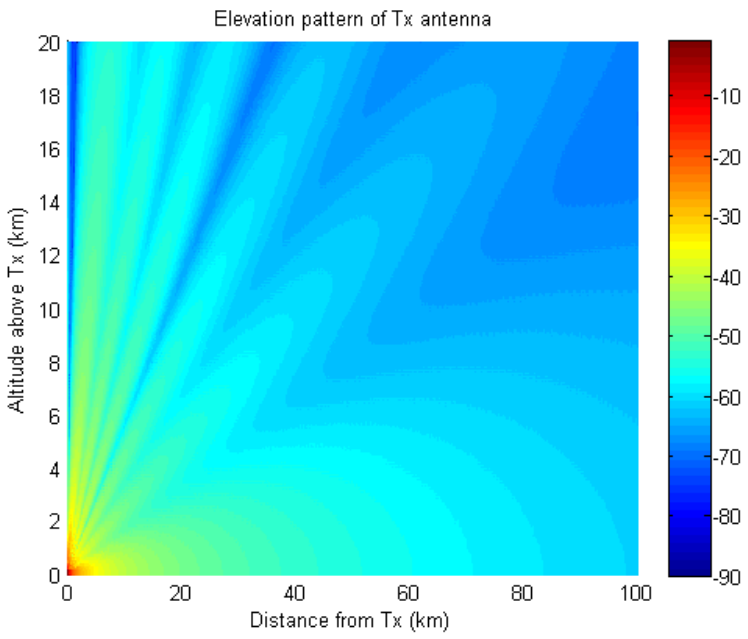

Fig. 10. Transmitter elevation power-density plot of a 6-bay FM antenna.

From Fig. 10 it is clear that a target at constant flight-level (e.g., $10 \mathrm{~km}$ ) may traverse multiple sidelobes, nulls, and the mainlobe during flight, leading to the aforementioned SNR fluctuations of the observed target.

An FM radio 8-bay antenna has an even narrower elevation mainlobe-width due to the greater number of vertical stacks in the array ( 8 instead of 6$)$. Fig. 11 shows the elevation pattern of an 8-bay FM radio transmit antenna.

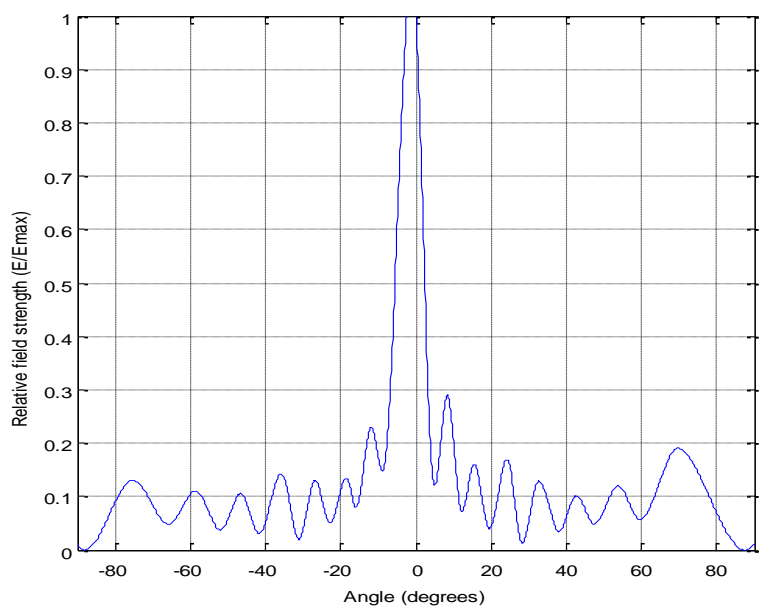

Fig. 11. Elevation pattern of an 8-bay FM radio transmit antenna. In this case the beam-tilt angle is $1^{\circ}$ with a directivity of $9.31 \mathrm{dBd}$.

The elevation pattern in Fig. 11 is typical of a main station site covering a significant population. In this case the depression angle is $1^{\circ}$ and the elevation HPBW is $6.8^{\circ}$. The relative power level of the 8-bay FM radio antenna is plotted in Fig. 12 $[16,17]$.

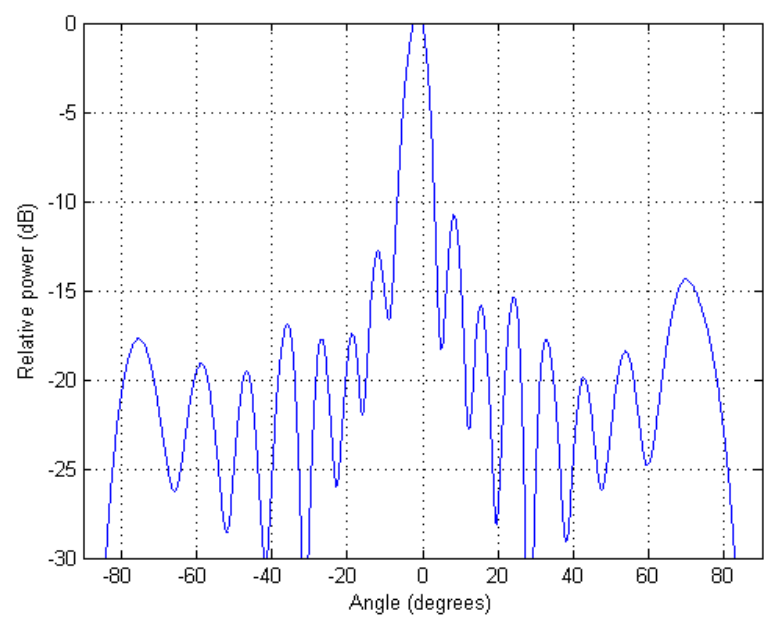

Fig. 12. Elevation pattern of an 8-bay FM radio transmit antenna in logscale.

For a depression angle of $1^{\circ}$, the angular width of the mainlobe above the $0^{\circ}$ line is approximately $2.4^{\circ}$ at the elevation HPBW point. 
Fig. 13 shows the 8-bay FM radio elevation pattern incorporated into a power-density plot $\left(\mathrm{dBW} / \mathrm{m}^{2}\right)$ to illustrate the power fluctuations throughout the pattern.

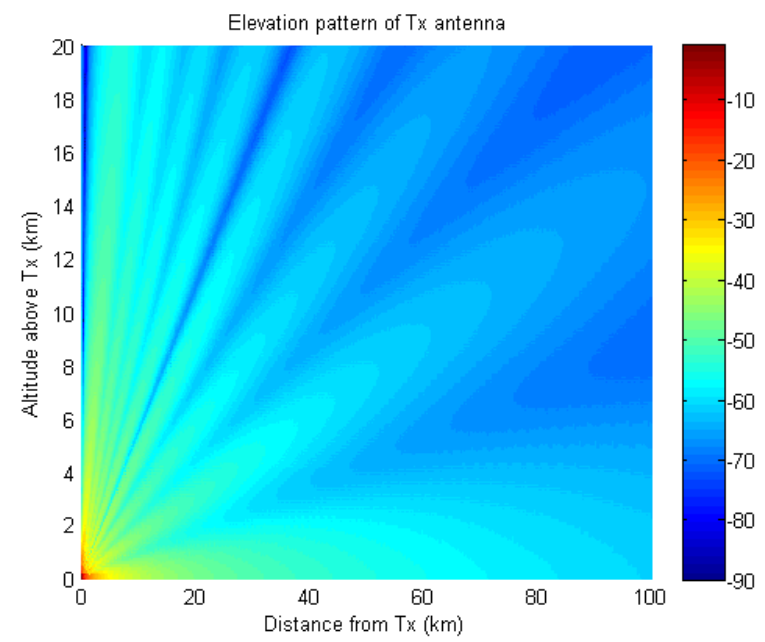

Fig. 13. Transmitter elevation power-density plot of an 8-bay FM antenna.

Once again it can be seen from Fig. 13 that a target at constant cruising altitude would traverse multiple sidelobes and may not even be illuminated by the mainlobe. This plot was computed for a transmit power $\left(P_{T}\right)$ of $10 \mathrm{~kW}$. With the gain of the antenna, this would give and ERP of approximately 100 $\mathrm{kW}$ at the peak of the beam.

\section{DAB AND DVB-T BROADCAST ANTENNAS}

$\mathrm{DAB}$ and DVB-T antennas vary in configuration and most manufacturers have multiple options to offer, making it difficult to provide a standardised estimate of elevation coverage. The broadcast business is one where tailoring of the azimuth and elevation patterns of transmission sites is common. The elevation patterns shown in this paper are indicative of what may potentially be installed at broadcast sites around the world. The patterns provide a metric for approximating the elevation coverage of DAB and DVB-T based passive radars.

In many countries, DAB antennas are vertically polarised. The standalone $\mathrm{DAB}$ antennas are often vertical dipole arrays, or simply vertical panel arrays. As the antennas are vertically polarised the radiators are stacked at dimensions between $0.75 \lambda$ and $\lambda$. The larger element spacing usually results in significant grating lobes at large angles of elevation. Therefore, the vertical spacing is often adjusted to the minimum due to concerns of on-ground EMR levels. The vertical aperture occupied can range between $\lambda$ and $16 \lambda$, approximately, for the largest antennas [12].

\section{DAB ELEVATION COVERAGE}

Fig. 14 shows the elevation pattern of a 4-bay DAB antenna.
In this case the beam-tilt angle is $0.7^{\circ}$. The elevation HPBW is approximately $11^{\circ}$. For a beam-tilt of $0.7^{\circ}$, the angular width of the mainlobe above the $0^{\circ}$ line is approximately $4.8^{\circ}$ at the elevation HPBW point.

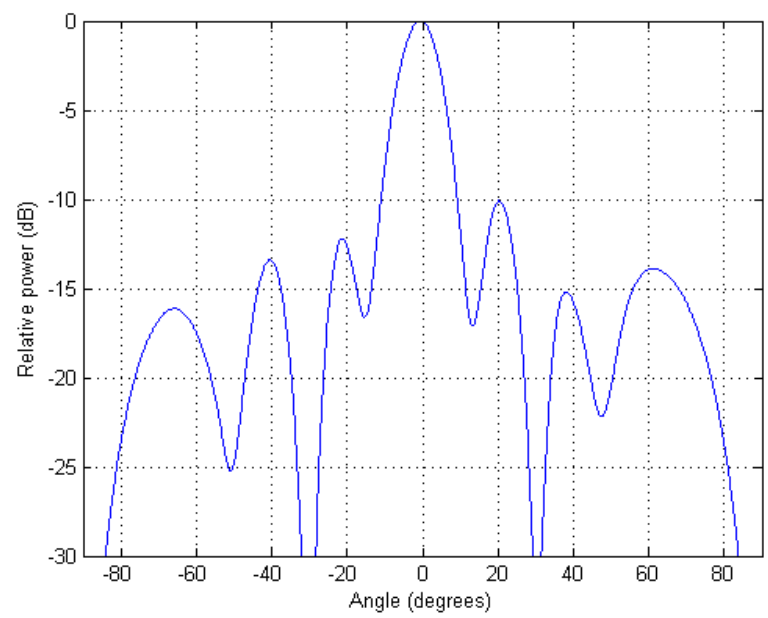

Fig. 14. Elevation pattern of a 4-bay DAB transmit antenna in log-scale.

Fig. 15 shows the 4-bay DAB elevation pattern incorporated into a power-density plot (in $\mathrm{dB}$ scale) to illustrate the power fluctuations throughout the pattern. As the ERP of DAB transmissions are generally lower than their $\mathrm{FM}$ radio and DVB-T counterparts, the DAB-based PBR detection range is also lower. As a result, Fig. 14 is plotted over a $50 \mathrm{~km}$ range extent.

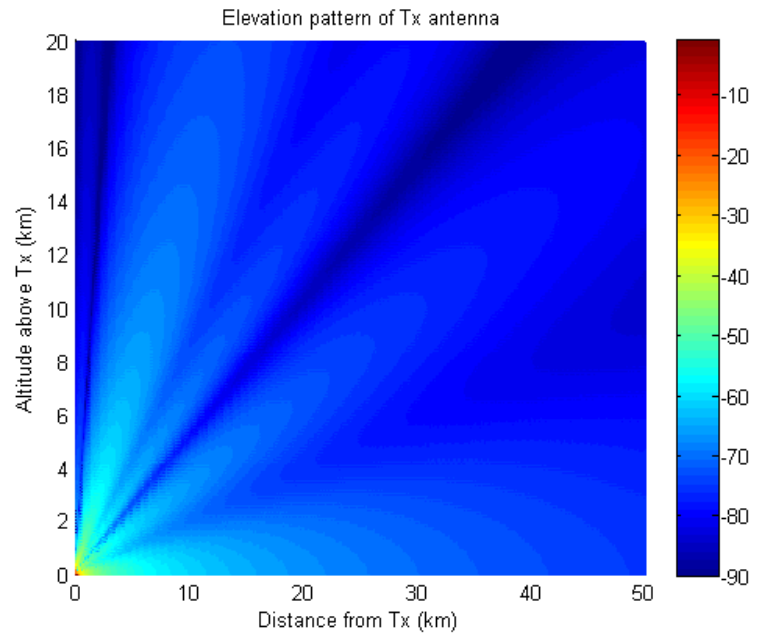

Fig. 15. Transmitter elevation power-density plot of a 4-bay DAB antenna. This plot has been computed for a transmit power $\left(P_{T}\right)$ of $1 \mathrm{~kW}$.

A DAB 8-bay antenna exhibits an even narrower elevation mainlobe-width due to twice as many vertical stacks in the array. Fig. 16 shows an elevation pattern of an 8-bay DAB transmit antenna. 


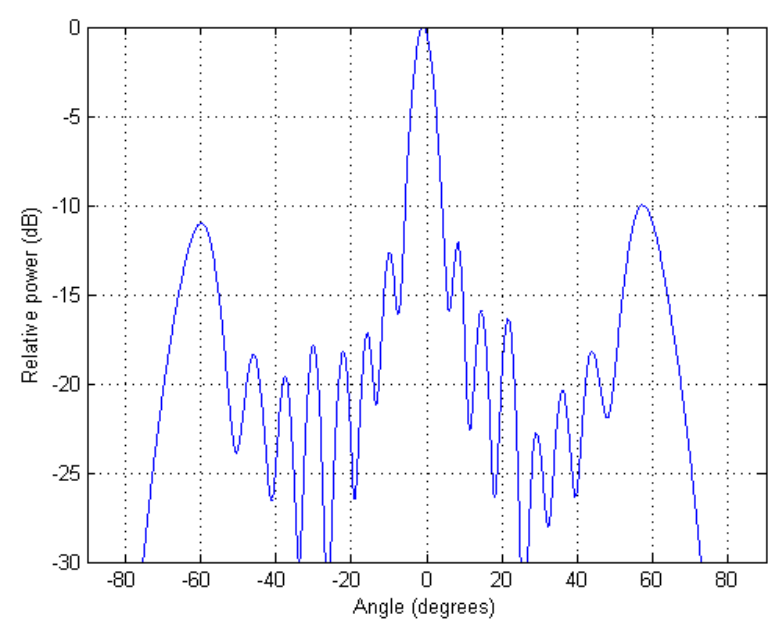

Fig. 15. Elevation pattern of an 8-bay DAB transmit antenna in log-scale.

The field pattern at the elevation HPBW in Fig. 16 is approximately $5.6^{\circ}$. For a beam-tilt angle of $1^{\circ}$, the angular extent of the mainlobe above the $0^{\circ}$ line is approximately $1.8^{\circ}$ at the elevation HPBW point.

Fig. 17 shows the 8-bay DAB elevation pattern incorporated into a power-density plot $\left(\mathrm{dBW} / \mathrm{m}^{2}\right)$.

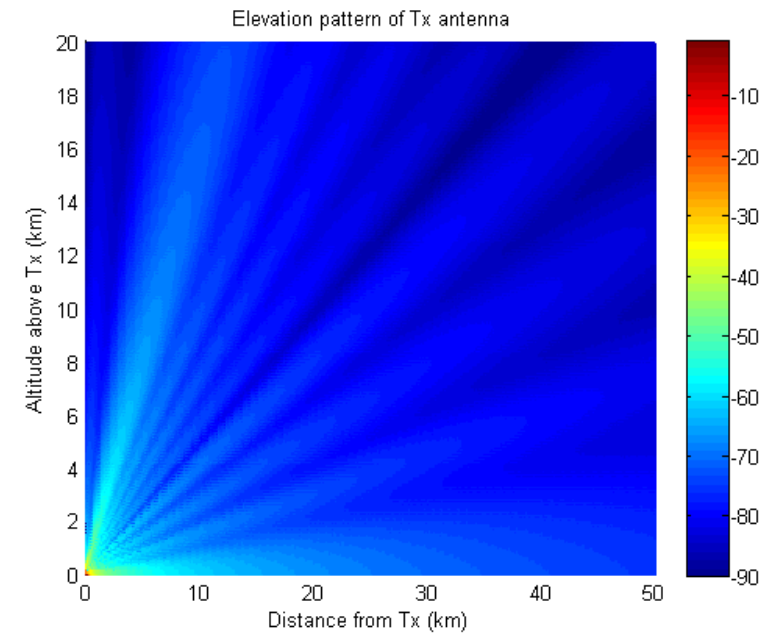

Fig. 17. Transmitter elevation power-density plot of an 8-bay DAB antenna. This plot has been computed for a transmit power $\left(P_{T}\right)$ of $1 \mathrm{~kW}$.

\section{DVB-T ELEVATION COVERAGE}

Digital Video Broadcast-Terrestrial, DVB-T, operates between $450 \mathrm{MHz}$ and $900 \mathrm{MHz}$. The physical size of the antenna elements at UHF is obviously smaller than those of FM radio and DAB. Therefore a greater number of DVB-T elements can be stacked on a transmitter mast to achieve a narrow elevation mainlobe shape and beam-tilt angle.

DVB-T antennas are of two broad types. The first type is panel antennas in which the main building block is a $2 \lambda$ panel which has four dipoles in front of a flat screen. The dipoles are spaced vertically at half wavelength spacing and as such have low radiation up and down the vertical aperture of the antenna and good gain characteristics. Panel arrays will normally consist of multiple panels around a support structure. For omnidirectional antennas some manufacturers provide/install arrays which have between 4 and 16 panels around the mast. The number of panels around is dictated by a number of factors including input power, access requirements, horizontal radiation pattern and the tower or mast section that the antenna may be required to be mounted on. Panel antennas allow extensive tailoring of the horizontal and elevation patterns. The amount of vertical aperture occupied with these antennas varies from $2 \lambda$ up to $32 \lambda$ (16 panels high) [16].

The other main DVB-T antenna types are slots, turnstiles, and similar radiating platforms where the radiators are spaced at wavelength spacing. These typically have larger grating lobes at high angles of elevation [16].

The antenna elevation pattern in Fig. 17 is from an 8-bay UHF panel array with a vertical spacing of $1.15 \mathrm{~m}$ between array elements.

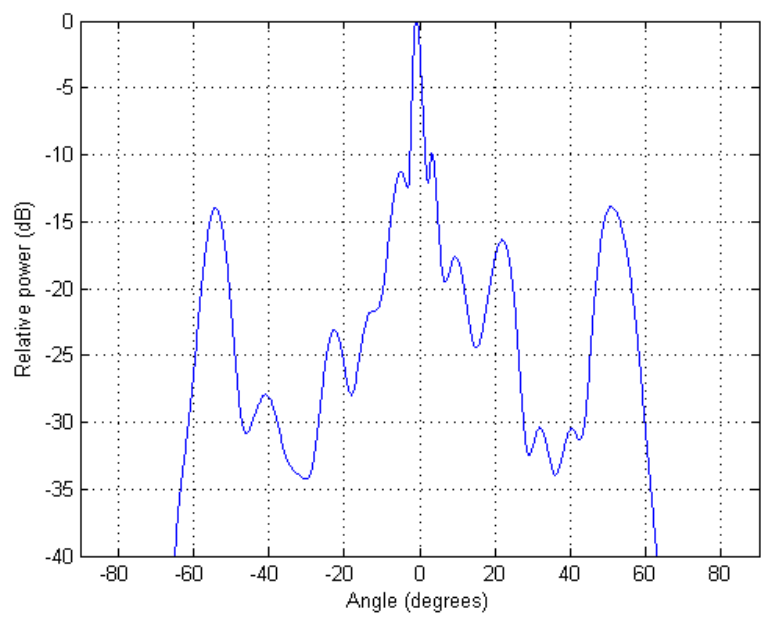

Fig. 18. Elevation pattern of an 8-bay DVB-T transmit antenna in log-scale.

The field pattern at the elevation HPBW in Fig. 18 is approximately $3^{\circ}$. For a depression angle of $1^{\circ}$, the angular extent of the mainlobe above the horizon line is $0.5^{\circ}$ at the elevation HPBW point.

Most targets at medium- and cruising-altitudes would be outside of mainlobe illumination and would rather be illuminated by one of the lower sidelobes.

Fig. 20 shows the 8-bay DVB-T elevation pattern incorporated into a power-density plot to illustrate the power fluctuations throughout the pattern. 


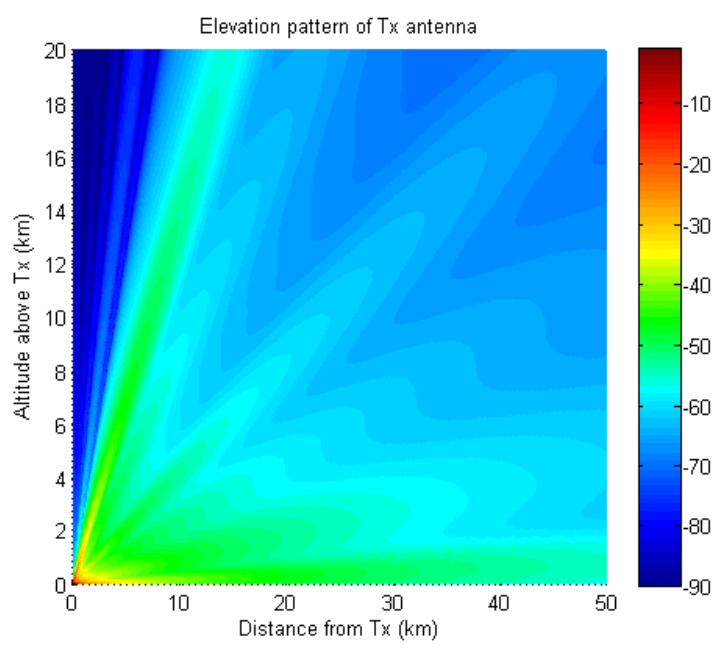

Fig. 20. Transmitter elevation power-density plot of an 8-bay DVB-T antenna. This plot has been computed for a transmit power $\left(P_{T}\right)$ of $10 \mathrm{~kW}$.

\section{AVAILABLE SNR AT GIVEN ALTITUDES ABOVE THE MAIN ILLUMINATORS OF OPPORTUNITY}

Fig. 21 shows power density fluctuation with the sine of the elevation angle (between 0 and 1) for three different elevation patterns. The pattern peak power has been normalised to $0 \mathrm{~dB}$. It can be seen that the antennas of the VHF FM radio transmitters have relatively high sidelobes, but the array of the DVB-T transmitter allows greater control of the radiation pattern and hence lower sidelobes.

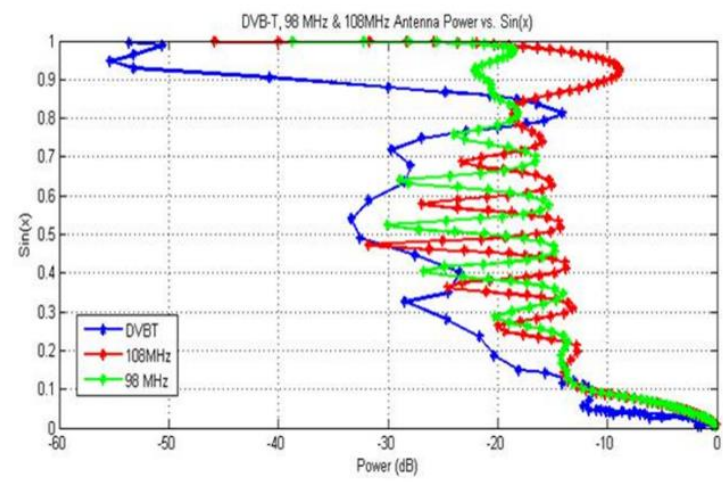

Fig. 21. Measured vertical-plane radiation patterns of BBC VHF FM radio transmitter at $98 \mathrm{MHz}$ (green), $108 \mathrm{MHz}$ (red) and 8-bay DVB-T transmitter (blue). The vertical scale is the sine of the elevation angle at the transmitter.

We provide now specific examples of $S N R$ fluctuations with elevation for FM radio, DAB and DVB-T that are indicative of typical PBR illuminator of opportunity considerations throughout the world. In bistatic radar it is common practice to plot the ovals of Cassini (constant sensitivity contours for a Swerling 5 point target) as a function of the available signalto-noise power ratio $(S N R)$. The Cassini ovals are routinely plotted in a horizontal/azimuth plane and illustrate $S N R$ variations at distances from the transmitter and receiver. The ovals can also be used to determine the constant SNR contours in the vertical/elevation plane. This is given in (3):

$$
\begin{aligned}
& S N R=\frac{P_{T} G_{T} G_{R} \lambda^{2} \sigma_{B}}{(4 \pi)^{3} R_{T}^{2} R_{R}^{2} k T_{S} B} \\
& P_{T}=10 \mathrm{~kW} \\
& G_{T}=\text { relevant antenna elevation pattern } \\
& G_{R}=1 ; \text { receiver assumed omnidirectional } \\
& \lambda \quad=3 \mathrm{~m}(\mathrm{FM}) ; 1.33 \mathrm{~m}(\mathrm{DAB}) ; 0.44 \mathrm{~m}(\mathrm{DVB}-\mathrm{T}) ; 1 \mathrm{~m} \\
&(\text { omnidirectional) } \\
& \sigma_{B}=\text { target bistatic radar cross section } \rightarrow \text { assumed here } \\
& 10 \mathrm{~m}^{2} \\
& k T_{S}=1.38 \times 10^{-23} \mathrm{~J} / \mathrm{K} \times 300 \mathrm{~K}=4.14 \times 10^{-21} \mathrm{~J} \\
& B=\text { a fixed bandwidth of } 2 \mathrm{MHz} \text { has been used for each } \\
& \text { system to yield a consistent noise power }(N) . \text { In } \\
& \text { practice, FM radio, DAB and DVB-T based passive } \\
& \text { radars will each have different system bandwidths. The } \\
& \text { noise power, } N, \text { is therefore } 8.28 \times 10^{-15} \mathrm{~J} / \mathrm{s}(-141 \\
& \text { dBW). }
\end{aligned}
$$

Fig. 22 shows plots of the $S N R$ variation on a scale from 25 dB to $-35 \mathrm{~dB}$. The plots shows the FM radio 8-bay, DAB 8bay and the DVB-T 8-bay antennas. Three altitude ranges are plotted, they are (from left to right): $2000 \mathrm{~m}, 5000 \mathrm{~m}$ and $10000 \mathrm{~m}$ (approximately cruising altitude). The lower plots in Fig. 22 provide an omnidirectional reference at the three considered altitude ranges.

In each figure of Fig. 22 the transmitter is located at $(20 \mathrm{~km}$, $20 \mathrm{~km})$ and the receiver is located at $(40 \mathrm{~km}, 20 \mathrm{~km})$. The baseline length is therefore $20 \mathrm{~km}$, and has been chosen as a realistic distance for the purpose of this simulation. The heights of the transmitter and the receiver have both been set to $50 \mathrm{~m}$, however, in reality there will be a difference between the two. Setting both to the same height results in an intensity plot that is a function of only the transmitter elevation pattern variation, which is the aim of this illustrative simulation.

The radar parameters of (3) have been kept identical for the three test cases of FM radio, DAB and DVB-T. Therefore, Fig. 22 is a function of increasing range only, that is, $S N R \propto$ $1 / R_{T}^{2} R_{R}^{2}$ (in this case the altitude above the Tx-Rx pair). As the transmitter elevation patterns have shape (i.e., are not omnidirectional) it is informative to observe their pattern differences between. It can be seen that the FM and DAB antenna patterns are approximately similar in shape, however, the FM radio pattern has an elevation HPBW of $6.8^{\circ}$ while the DAB has $5.6^{\circ}$. Therefore, the FM radio 8-bay antenna provides slightly superior illumination coverage at higher elevations than the corresponding DAB antenna. This may be contrasted to the omnidirectional plots where the antenna pattern is uniform in space: In the FM radio and DAB plots it can be seen that as the target altitude increases, the highest SNR regions become more localised around the receiver site. The omnidirectional plots, in contrast, always remain symmetrical with no greater or lesser intensity around the receiver site (an obvious outcome).

The DVB-T elevation patterns, due to their narrow mainlobe of approximately $3^{\circ}$, exhibit a very poor capability of being able to illuminate targets at medium and cruising 
altitudes at close ground range to the transmitter. It can be seen that for a target altitude of $10000 \mathrm{~m}$, a large sector of the geometrical surface is not illuminated at a level of $-35 \mathrm{~dB}$. This result corroborates experimental measurements that DVB-T has severe limitations for target detection at mediumand cruising-level altitudes, especially for targets at closer range to the transmitter.

\section{Conclusion}

Bistatic/multistatic radars using broadcast illuminators of opportunity have elevation mainlobes that are tilted below the horizon. Their "natural ability" therefore is not ideal for high altitude airspace illumination at ranges nearer the transmitter. The down-tilt profile does, however, yield advantages for illuminating targets when they first appear above the horizon at medium to far ranges from the transmitter. This is a coverage advantage where PSRs are least capable - that is, illuminating the low altitude airspace are farther ranges from the radar. It is important to realise, though, that illuminators of opportunity often do detect higher- and cruising-altitude targets because of the portion of radiated power above and beyond the horizon (see (2)). Bistatic Radar detection SNR values could also potentially be higher for cruising altitude targets than in certain PSR scenarios because the bistatic receiver could be located closer to the target. That is, the scattering path from target to receiver could be shorter than the scattering path from the target to the monostatic PSR.

Nevertheless, as has been remarked throughout, the elevation patterns of broadcast antennas exhibit strong lobing. As targets travel, even at constant flight-level, they traverse multiple sidelobes and nulls. The energy returned to the receiver fluctuates and may result in tracks being lost. From the elevation patterns it can also be seen that the pattern sidelobes are not symmetric, often due to the process of nullfilling.

The lobes present in broadcast elevation patterns are also in contrast with the elevation patterns of 2-D surveillance radars employing $\operatorname{cosec}^{2}$ beams. Surveillance radars attempt to reduce high elevation lobing to prevent track degradation. As shown in Fig. 4, PSRs sometimes use dual-beam antenna patterns to improve the detection of short-range targets in competition with clutter [18]. This is not a feature of illuminators of opportunity.

This paper has compiled the elevation patterns of FM radio, DAB and DVB-T illuminators of opportunity. Although the principles of mainlobe shaping and beam-tilt are widely known, their impact on Passive Radar performance has not been characterised in detail. This work has attempted to provide greater detail on broadcast illuminator elevation characteristics and how they are important in Passive Radar performance.

\section{ACKNOWLEDGEMENTS}

The authors gratefully acknowledge the invaluable input and assistance of Kevin Thompson, RFS Australia. The data and information provided by the antenna manufacturer, RFS, has added validity to the present material, which the authors hope will be a useful resource for other practitioners working with FM radio, DAB and DVB-T illuminators. We are also extremely grateful for the insights and technical input of Dr. Charles Williams, Gadzooks Australia, for sharing his wealth of insight. During his employment with RFS, Dr. Williams and his colleagues were honored with an Emmy Award for their pioneering work on 'adjacent channel combiners' for TV broadcasting.

\section{REFERENCES}

[1] R. C. Dorf, "The Electrical Engineering Handbook $2^{\text {nd }}$ Edition," $C R C$ Press, 1997, pp. 1605.

[2] J. J. Carr, "Practical Antenna Handbook $4^{\text {th }}$ Edition," McGraw-Hill, 2001.

[3] Author's personal communications with Fraunhofer FHR test pilot.

[4] Author's personal communications with recently retired Luftwaffe combat pilot (rank upon retirement: Major).

[5] G. W. Stimson, "Introduction to Airborne Radar $2^{\text {nd }}$ Edition," SciTech Publishing, New Jersey, 1998.

[6] "Multilateration \& ADS-B Executive Reference Guide," published with support from ERA.

[7] F. Colone, D. W. O'Hagan, P. Lombardo and C. J. Baker, “A multistage processing algorithm for disturbance removal and target detection in passive bistatic radar," IEEE Transactions on Aero. \& Elect. Sys., Vol 45, No. 2, April 2009, pp. 698-772.

[8] D. W. O'Hagan and C. J. Baker, "Passive bistatic radar (PBR) using FM radio illuminators of opportunity," IEEE Passive '08, France, Oct. 2008, pp. 1-6.

[9] $3^{\text {rd }}$ PCL Focus Days, Fraunhofer-FHR, Wachtberg, Germany, May 2011.

[10] H. Kuschel, J. Heckenbach, D. W. O'Hagan, M. Ummenhofer, "Exploitation of DVB-T signals for passive radar," Fraunhofer-FHR Bericht No. 146, March 2010.

[11] P. Howland, D. Maksimiuk, G. Reitsma, "FM radio based bistatic radar," IEE Proc. Radar Sonar Navig., Vol. 152, No. 3, June 2005, pp. $107-115$.

[12] Indra, "Primary Surveillance Radar", http://www.indracompany.com

[13] UK Civil Aviation Authority (CAA), "Air Traffic Services Safety Requirements CAP 670", May 2014.

[14] Author's personal communications with Dr. C. Williams, Gadzooks Australia.

[15] The BBC Engineering Division, "Shaping the vertical radiation patterns of VHF and UHF transmitting aerials by phase perturbation", Research Report E-121, December 1966, pp. 1 - 28.

[16] Author's personal communications with RFS World.

[17] Author's personal communications with Kathrein Antennas.

[16] D. K. Barton, Radar System Analysis and Modeling, Artech House, Boston, 2005. 

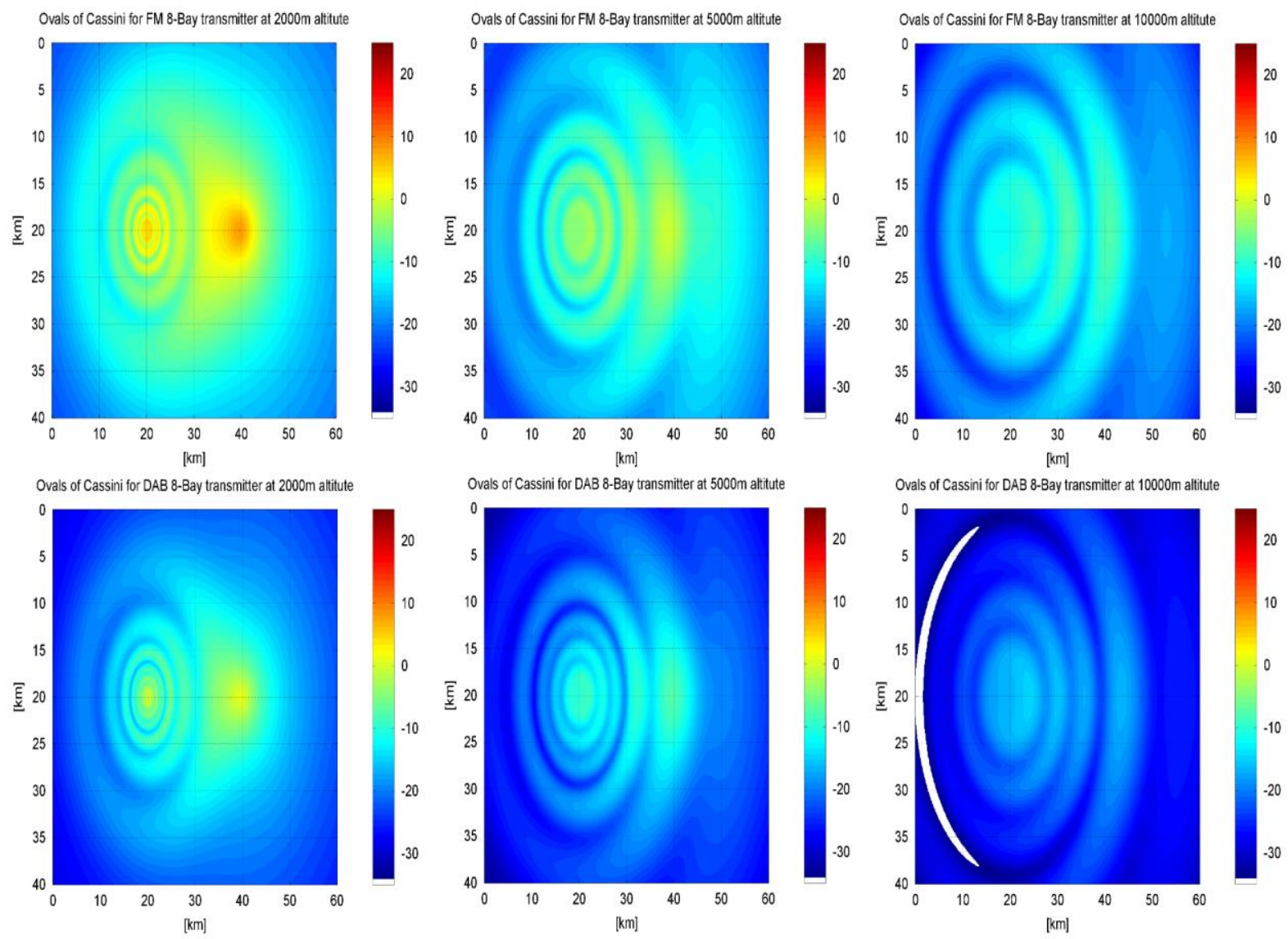

Ovals of Cassini for DAB 8-Bay transmitter at $5000 \mathrm{~m}$ alitute
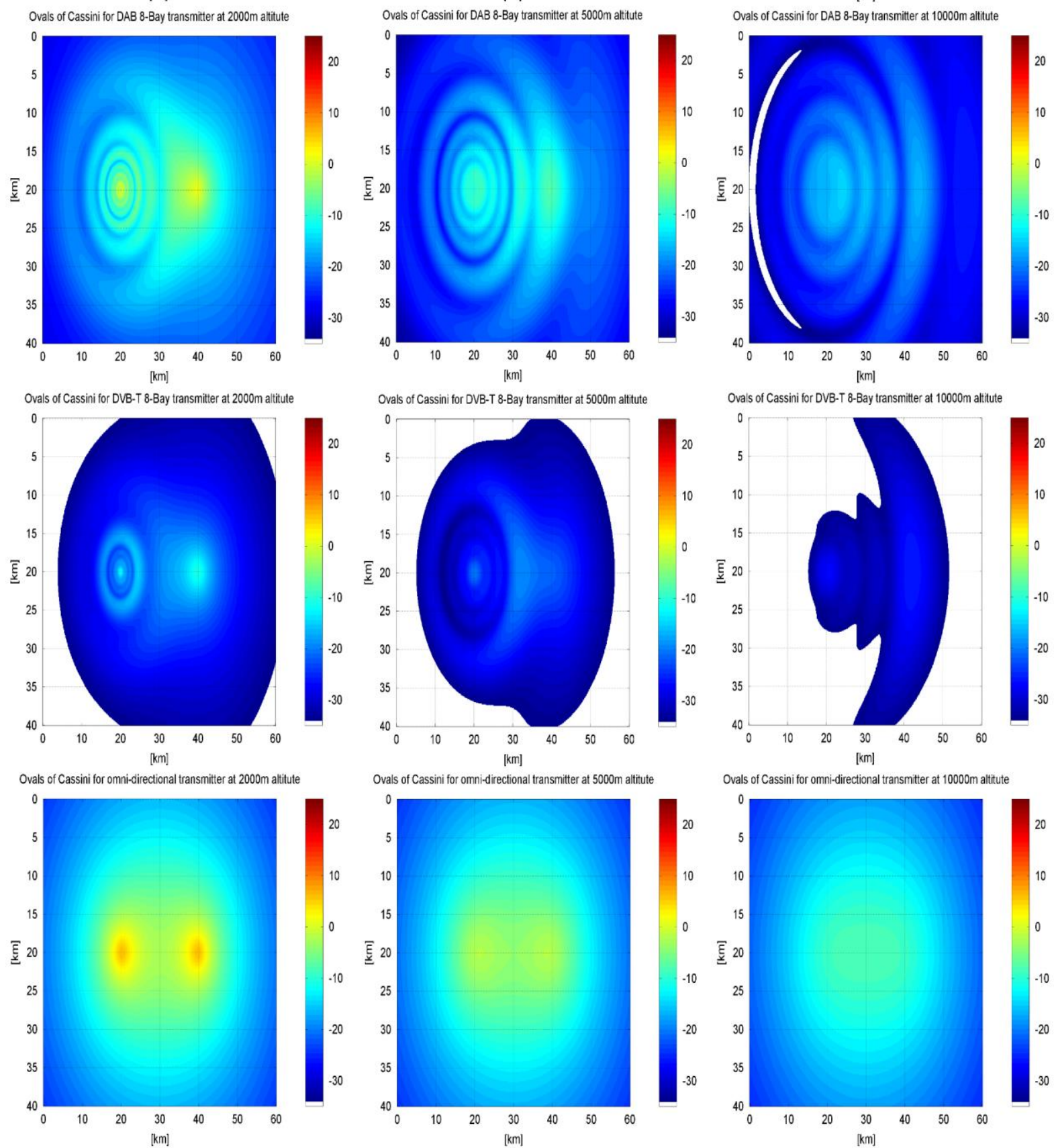

Fig. 22. Ovals of Cassini plotted in the elevation plane for altitudes of $2000 \mathrm{~m}, 5000 \mathrm{~m}$ and $10000 \mathrm{~m}$. FM radio, DAB and DVB-T patterns are plotted against an omnidirectional reference. 